\title{
PAPR Reduction in LTE Down Link using a Hybrid Repeated Clipping and SQRT Companding
}

\author{
Zainab S. Hadi \\ University of Baghdad, College of Engineering \\ Electronic \& Communications Engineering \\ Department
}

\author{
Buthaina M. Omran, Ph.D \\ University of Baghdad, College of Engineering \\ Electronic \& Communications Engineering \\ Department
}

\begin{abstract}
The Orthogonal Frequency Division Multiplexing is one of the widely used modulation techniques in the broadband wireless technology. One of the main problems of the OFDM is the high peak-to-average power ratio of transmitting signal due to the superposition of many subcarriers. This paper presents a new hybrid peak-toaverage power ratio reduction technique, which repeated clipping and frequency domain filtering with SQRT companding over LET channel. The paper highlights the performance and advantages of the mixed technique. The simulations show that the proposed technique realizes an improved PAPR With acceptable BER.
\end{abstract}

\section{Keywords:}

Complementary cumulative distribution function (CCDF), OFDM, peak-to-average power ratio (PAPR), SQRT companding, repeated clipping and filtering.

\section{INTRODUCTION}

The basic principle of OFDM is to split a high data rate streams into a number of lower data rate streams and then transmitted these streams in parallel using several orthogonal sub-carriers (parallel transmission). When the subcarriers have appropriate spacing to satisfy the orthogonality, their spectra will overlap [1]. OFDM has been adopted in many wireless communication standards, including European digital audio broadcasting, terrestrial digital video broadcasting and satellite-terrestrial interactive Multiservice infrastructure in China. In addition, OFDM has been considered and approved by many IEEE standard working groups, such as IEEE $802.11 \mathrm{a} / \mathrm{g} / \mathrm{n}$, IEEE $802.15 .3 \mathrm{a}$, and IEEE $802.16 \mathrm{~d} / \mathrm{e}$ [2 - 3]. The applications of OFDM include wireless personal area networks, wireless local area networks, and wireless metropolitan networks. Currently, Orthogonal Frequency Division Multiple Access (OFDMA) is being investigated as one of the most promising radio transmission techniques for LTE of the 3rd Generation Partnership Project (3GPP), International Mobile Telecommunications-Advanced Systems [4].

OFDM offers many advantages over single-carrier modulations but high PAPR of transmitting signals is one of the major issues of the OFDM system. A large dynamic range of input data symbols is the main cause of getting high PAPR. An OFDM signal consists of $N$ independent data symbols modulated on $\mathrm{N}$ orthogonal subcarriers, and when these $\mathrm{N}$ signals are added to the same phase, higher peak amplitude is observed. The value of this peak may be $\mathrm{N}$ times of the average amplitude [5].

The drawback of a large dynamic range is that it places pressure on the design of components such as the word length of the IFFT/FFT pair, mixer stages, and most importantly the HPA, which must be designed to handle irregularly occurring large peaks, decreases the SQNR (Signal-to-Quantization Noise Ratio) of ADC (Analog-toDigital Converter) and DAC (Digital-to-Analog Converter). Failure in design components with a sufficiently large linear range result in saturation of the HPA. Saturation creates both in band distortion, increasing the BER and out of band distortion, or spectral splatter, which causes ACI. One obvious solution is to design the components to operate within large linear regions, however this is impractical as the components will be operating inefficiently and the cost becomes prohibitively high. This is especially apparent in the HPA where much of the cost and $\sim 50 \%$ of the size of a transmitter lies $[6,7]$.

Many PAPR reduction schemes based on different techniques, such as clipping and filtering, window shaping, block coding, partial transmit sequence (PTS) technique [8], and selective mapping (SLM) technique [9], phase optimization, tone reservation [10] and injection [11], and nonlinear companding transform schemas. However, no specific PAPR reduction technique is the best solution for the OFDM system. Various parameters like loss in data rate, transmit signal power increase, BER increase, computational complexity increase should be taken into consideration before choosing the appropriate PAPR technique $[12,13]$.

\section{DEFINITION OF PAPR}

The PAPR is defined as the ratio between the maximum instantaneous power and its average power. If $x(t)$ is a transmitted baseband OFDM signal, then PAPR is defined as:

$\operatorname{PAPR}[\mathrm{x}(\mathrm{t})]=\frac{\left.\left.\left.\underset{0 \leq t \leq \mathrm{T}_{\mathrm{S}}}{\mathrm{MAX}_{\mathrm{S}}}\right|_{\mathrm{x}(\mathrm{t})}\right|^{2}\right]}{\mathrm{p}_{\mathrm{av}}}$

Where, $\mathrm{p}_{\mathrm{av}}$ Is the average power of $\mathrm{x}(\mathrm{t})$ and can be computed in frequency domain because IFFT is a unitary transformation $\mathrm{T}_{\mathrm{s}}$ Is useful duration of an OFDM symbol [14].

The above power characteristics can also be described in terms of their magnitudes (not power) by defining the crest factor $(\mathrm{CF})$ as:

$\mathrm{CF}=\sqrt{\mathrm{PAPR}}$ 


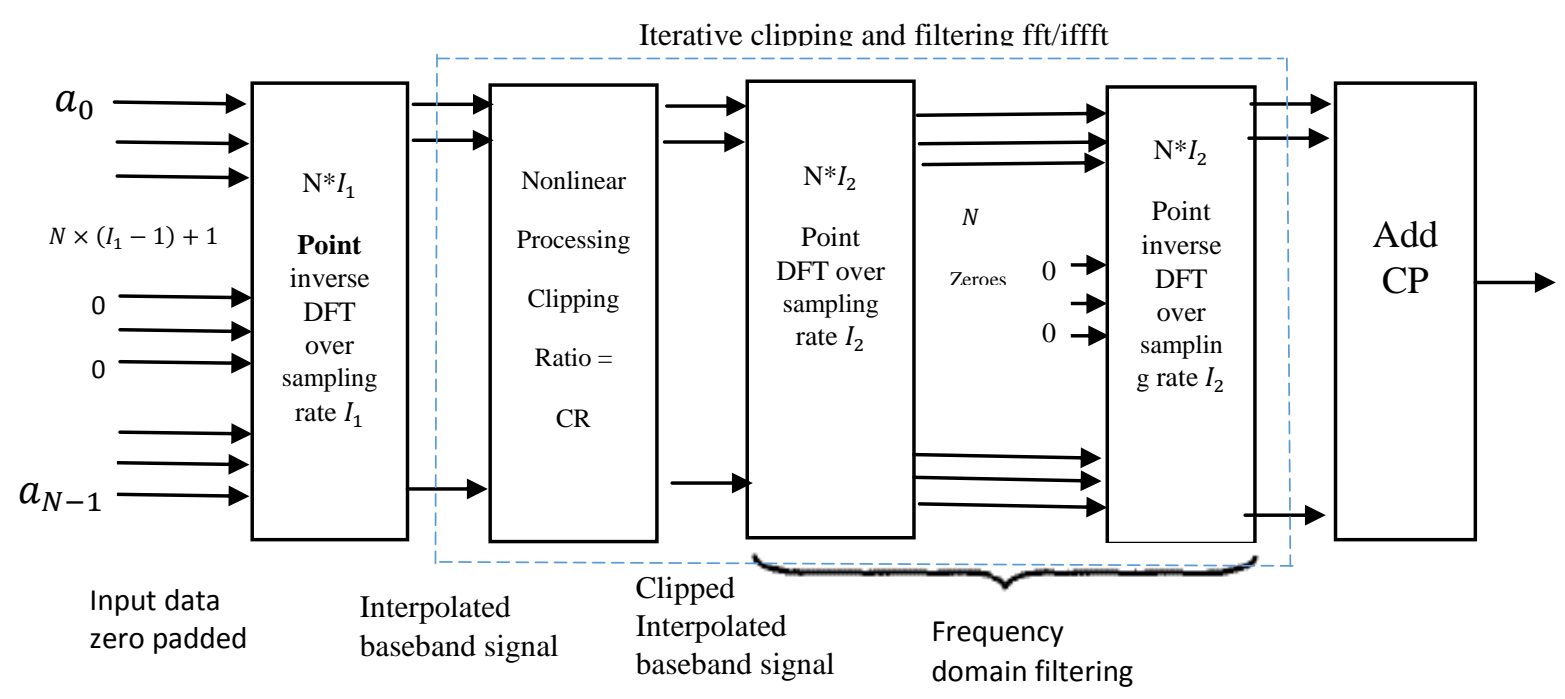

Fig 1: Clarifies the clipping and filtering process

\section{REPEATED CLIPPING AND FREQUENCY DOMAIN FILTERING}

In the clipping technique hard limiting is applied to the amplitude of the complex values of the IFFT output. The filtering technique is designed to alleviate or cancel out-ofband distortion dependent on oversampling value, but cannot correct in-band distortion [16]

At first the input data signal $\mathrm{a}_{0}, \ldots, \mathrm{a}_{\mathrm{N}-1}$ as shown in figure (1) is oversampled by a factor I by placing N (I - 1) zeroes in the middle of the vector. This oversampled signal is transformed into the time domain using an IFFT. This results in trigonometric interpolation of the time domain signal.

After an IFFT, the original signal is clipped in the time domain. The clipping can be described as shown below:

$$
\mathrm{C}=\left\{\begin{array}{ll}
\sqrt{\mathrm{c}_{\mathrm{R}} * \mathrm{E}\left[|\mathrm{x}|^{2}\right]} * \frac{\mathrm{x}}{|\mathrm{x}|} & |\mathrm{x}|^{2}>\mathrm{c}_{\mathrm{m}} \\
\mathrm{x} & |\mathrm{x}|^{2} \leq \mathrm{c}_{\mathrm{m}}
\end{array}\right\}
$$

Where $\mathrm{C}$ represents the output of the time domain signal $\mathrm{c}_{\mathrm{m}}=\mathrm{c}_{\mathrm{R}} * \mathrm{E}\left[|\mathrm{x}|^{2}\right], \mathrm{c}_{\mathrm{m}}$ Is the threshold clipping level signal power $=|x|^{2} ; E\left[|x|^{2}\right]$ Is the mean power $c_{R}$ The clipping ratio, is defined as the ratio of the clipping level to the mean power of the unclipped baseband signal The clipping is followed by frequency domain filtering to reduce out-of-band power.

The clipped time domain signal $\mathrm{c}$ is then converted back into the discrete frequency domain using an FFT, The in band discrete frequency components of the clipped signal $\mathrm{c}_{0}, \mathrm{C}_{\frac{\mathrm{N}}{2}-1}, \mathrm{c}_{\mathrm{NI}_{1}-\frac{\mathrm{N}}{2}+1}, \ldots, \mathrm{C}_{\mathrm{NI}_{1}-1}$ Are passed unchanged to the inputs of the second IFFT while the out-of-band components, $\mathrm{C}_{\frac{\mathrm{N}}{2}+1}, \ldots, \mathrm{c}_{\mathrm{NI}_{1}-\frac{\mathrm{N}}{2}}$ Are nulled [16]. This technique is repeated iteration number, usually choose between one and four.

The second IFFT is followed by serial to parallel conversion, digital to analogue conversion, modulation and amplification. Although frequency domain filtering is a common signal processing technique the form shown in figure (1) is unusual. In most filtering applications the filter is designed to meet particular specifications in the continuous frequency domain. In this application, the wanted signal is an OFDM signal, which is the sum of discrete frequency components in each symbol period.

The filter must therefore have as little effect as possible on the in-band discrete frequency domain while attenuating as much as possible any out-of-band components. Because the filter operates on a symbol by symbol basis, there is no filtering across symbol boundaries and so no resultant intersymbol interference. The filtering does cause some peak regrowth. However, this is much less than for clipping before interpolation. [15, 17]

The clipping noise is added at the transmitter rather than the receiver. In fading channels this means that in general the clipping noise will cause less degradation in bit error rate than noise added in the channel since the clipping noise fades along with the signal.

\section{SQUARE-ROOT COMPANDING}

The block diagram of a typical OFDM system using the original SQRT technique for PAPR reduction is shown in Fig.2. By using the SQRT technique, the original OFDM output signals $x_{n}$ Is processed by (4) before they are converted into analog waveforms and amplified by the power amplifier

$\mathrm{x}_{\mathrm{n}}^{\prime}=\sqrt{\left|\mathrm{x}_{\mathrm{n}}\right|} * \mathrm{e}^{\mathrm{j} \emptyset_{\mathrm{n}}}$

Is the new OFDM signal, and $\emptyset_{\mathrm{n}}$ Is the phase of $\mathrm{x}_{\mathrm{n}}$ During the entire signal processing, the phases of the OFDM output signals $\emptyset_{\mathrm{n}}$ Are kept unchanged while only the amplitudes are treated and changed [18].

For the complex Gaussian distributed signals, such as OFDM output signals, SQRT process changes the Rayleigh distribution of these signals into a Gaussian-like, close to 


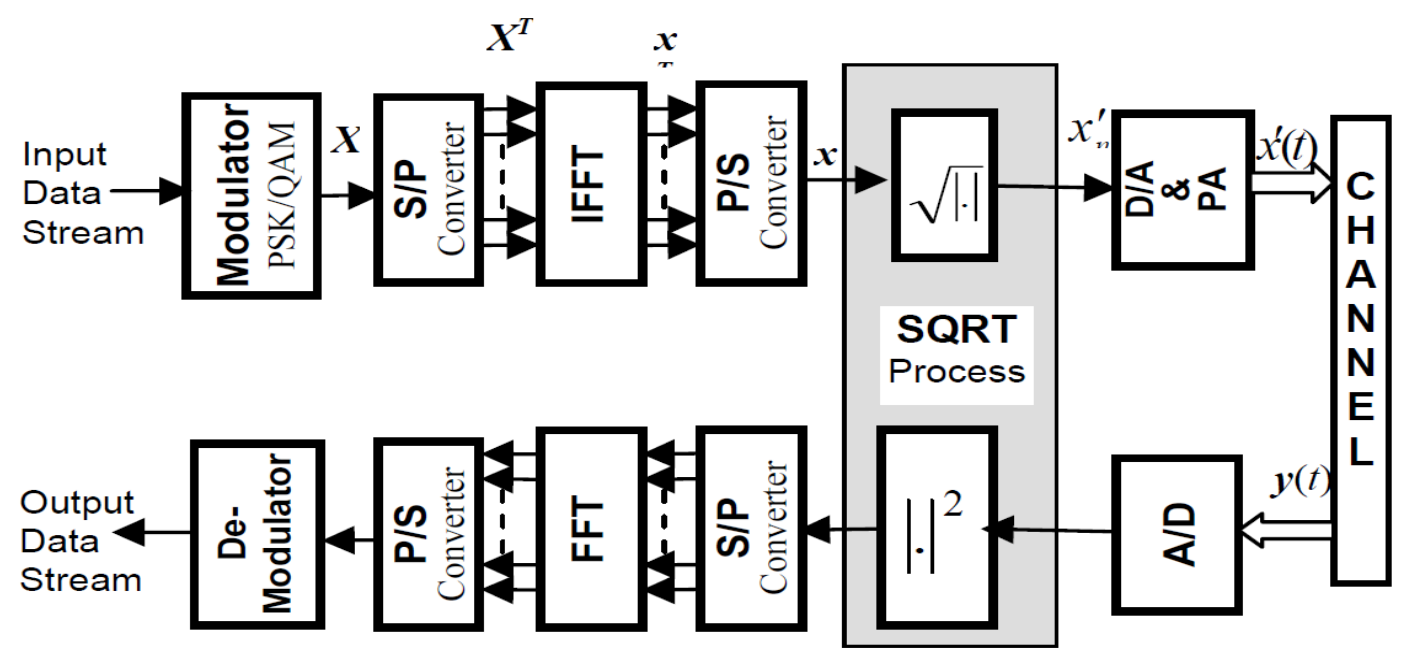

Fig 2: Block diagram of an OFDM system using SQRT technique

Gaussian, distribution [18,19]; while the Chi-square distribution is, converted, according to the analysis of these signals given in the previous section, to Rayleigh distribution. The latter is because the Rayleigh distribution in such signals represents voltage while the Chi-square distribution represents the power of the same signals. However, not only the statistical distribution is changed by the SQRT process, but the values of the mean $\mu$ and variance $\sigma^{2}$ of the processed OFDM output signals are also changing, and subsequently the values of the average power and peak power of these signals are altered also. To understand the effect of the SQRT process on the power values of OFDM output signals, we assume normalized average power (Pavg $=1)$. When the average power is normalized, the value of the peak power is diminished by $\mathrm{N}$ because Pavg $=\mathrm{N}$ for the same PAPR. This assumption is applicable for all OFDM symbols as the average power is constant and equal to $\mathrm{N}($ Pavg $=\mathrm{N})$. Hence, the PAPR can be analyzed according to (1) through the peak power only. The new value of normalized peak power is always greater than one because peak is constantly greater than Pavg in all OFDM symbols. Therefore, the SQRT process always causes a reduction in the value of the peak power of the normalized OFDM symbols, and as a result the PAPR is reduced in all sizes of OFDM blocks, N. In [18, 19] the SQRT process applies to the signals of all OFDM output symbols; therefore, the PAPR reduced without the need to send side information. The SQRT process changes the distribution of the power signals to Rayleigh distribution and reduces the value of average power from $\mathrm{N}$ to $\mathrm{N} 1 / 2$. The variance of the Rayleigh distribution equals $(2-\pi / 2) \sigma^{2}$ [20] which is approximately equal to half the value of variance of the Gaussian distributed signals. The SQRT process in the SQRT OFDM system performs this statistical transformation, and therefore results in a constant degradation in the BER rate equal to $3 \mathrm{~dB}$ because of decreasing of variance to the half of that of the conventional $\operatorname{OFDM} \operatorname{system}\left(\sigma_{\mathrm{SQRT}}^{2} \approx \frac{1}{2} \sigma_{\text {conv }}^{2}\right)$.

\section{THE PROPOSED PAPR REDUCTION METHOD}

We propose hybrid approach, which consist of repeated clipping and frequency domain filtering and SQRT compander as shown in figure (3). The main idea for combining the two methods is relaying on the observation that two different types of signal processing for PAPR reduction will increase the overall performance.

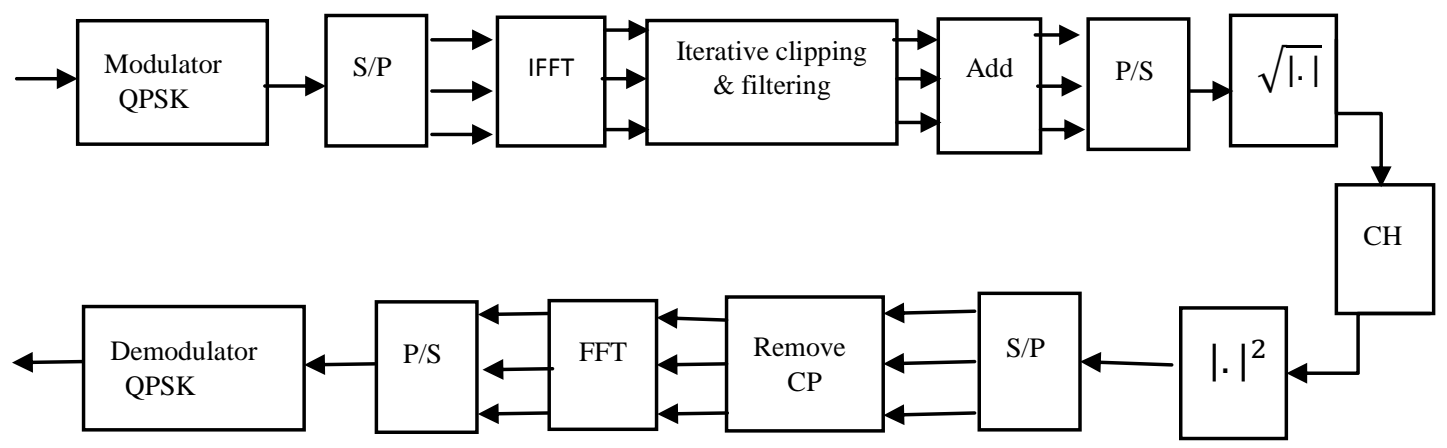

Fig 3: Proposed PAPR reduction 
Table 1. The value of PAPR and SNR at BER $=10^{-4}$

\begin{tabular}{|l|l|l|l|l|l|l|l|}
\hline PAPR & $\begin{array}{l}\text { SNR at BER } \\
=10^{-4}\end{array}$ & $\begin{array}{l}\text { PAPR } \\
\text { Clipping }\end{array}$ & $\begin{array}{l}\text { SNR at } \\
\text { BER }=10^{-4} \\
\text { Clipping }\end{array}$ & $\begin{array}{l}\text { PAPR } \\
\text { SQRT }\end{array}$ & $\begin{array}{l}\text { SNR at } \\
\text { BER }=10^{-4} \\
\text { SQRT }\end{array}$ & $\begin{array}{l}\text { PAPR } \\
\text { Hybrid }\end{array}$ & $\begin{array}{l}\text { SNR at } \\
\text { BER }=10^{-4} \\
\text { Hybrid }\end{array}$ \\
\hline 25.3766 & 11.872 & 14.5624 & 8.85 & 13.8413 & 14.466 & 8.4279 & 11.571 \\
\hline
\end{tabular}

\section{SIMULATION AND RESULT}

To verify the proposed PAPR reduction technique, we tested it in the LTE downlink system with parameters shown in Table 2

Table 2. Simulation parameter

\begin{tabular}{|l|l|}
\hline FFT size & 128 \\
\hline Spacing frequency & $15 \mathrm{KHz}$ \\
\hline BW & $1.25 \mathrm{MHz}$ \\
\hline CP & 32 \\
\hline No symbol & 1000 \\
\hline Sampling frequency & $192 \mathrm{MHz}$ \\
\hline $\begin{array}{l}\text { No. of path delay in LTE } \\
\text { channel }\end{array}$ & 6 \\
\hline
\end{tabular}

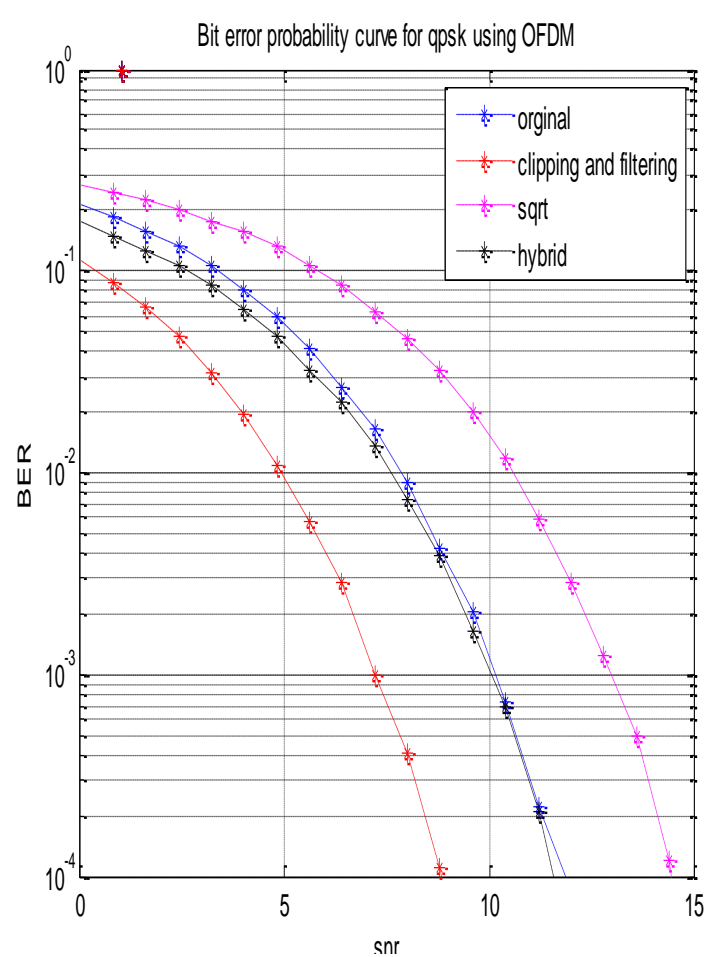

Fig 4: Comparison in BER between original signal, repeated clipping and frequency domain filtering, SQRT companding and proposed hybrid.

As shown in figure (4) the hybrid has better PAPR and BER compare, Hybrid result with oversampling equal to 2 and $\mathrm{CR}=4$, and the same thing for Just repeated clipping and frequency domain filtering, and we can see that repeated clipping and frequency domain filtering has the best BER because of filtering, While SQRT has worse BER but PAPR Slightly larger repeated clipping and frequency domain filtering.

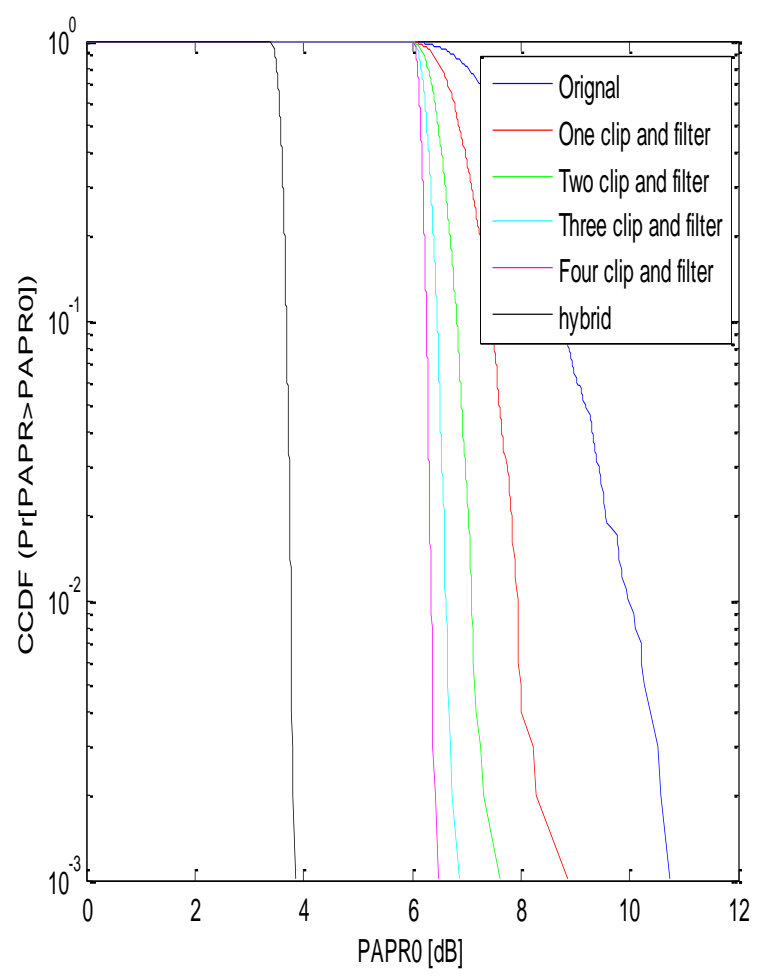

Fig 5: Comparison in PAPR between original signal, repeated clipping and frequency domain filtering and hybrid with $C R=4$, oversampling $=2$.

As shown in figure (5) there are about $6.887 \mathrm{~dB}(10.755$ $3.868=6.887$ ) Improvement in the PAPR value. Figure (5) is for PAPR after companding, but the total PAPR for original signal $=25.3766$ as shown in table $(1)$ for hybrid $=8$. $4279(25.3766-8.4279=16$. 9487) $16.9487 \mathrm{db}$ is the Improvement in the total PAPR. And with little improvement BER comparison with the original signal as shown in figure (4)

When we change I the Papr and BER will change because of frequency domain filtering that we use. We take $\mathrm{I}=1.125$, $1.25,1.5,2,3,4$ for explanation $\mathrm{I}=1.125$ that means $0.125^{*}$ FFT size of zero that add in the center input vector to make trigonometric interpolation. 


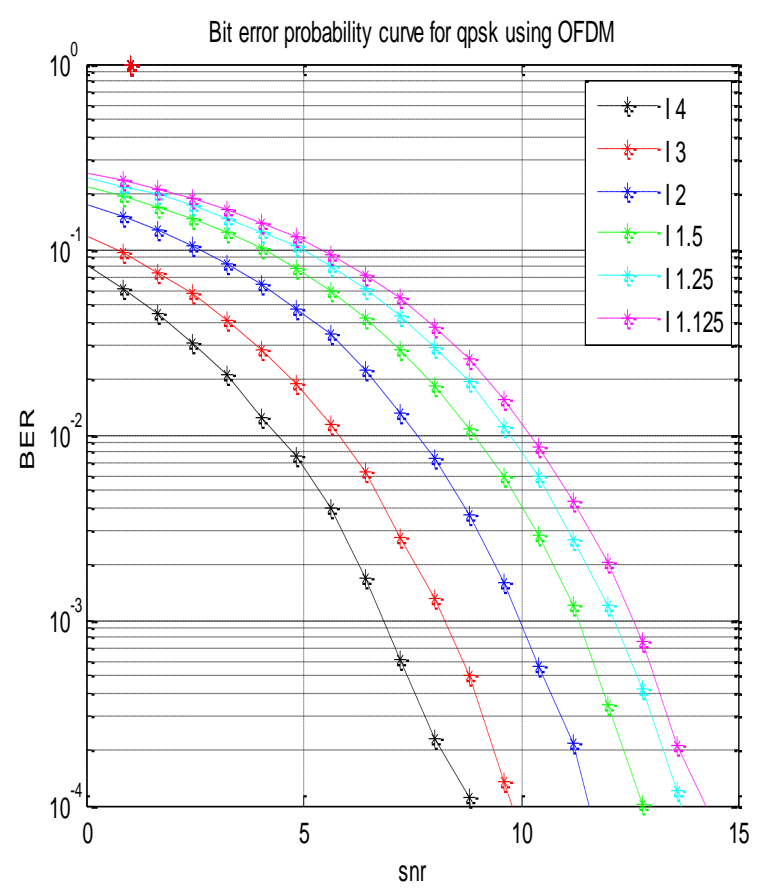

Fig 7: Comparison in BER for different oversampling value.

As shown in figure (6) and (7) an inverse relationship between BER and PAPR for oversampling $=4$ has a better BER but worse PAPR compare with other oversampling unlike oversampling $=1.125$, we can say that for large oversampling the BER improve, but PAPR Getting worse compare with oversampling and vice versa.

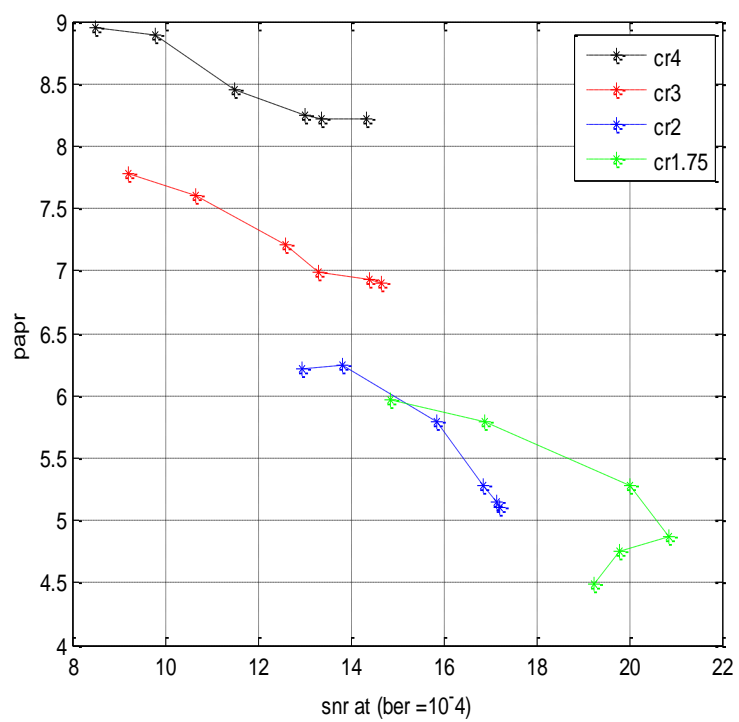

Fig 8: Relationship between PAPR and BER for different value of $C R$

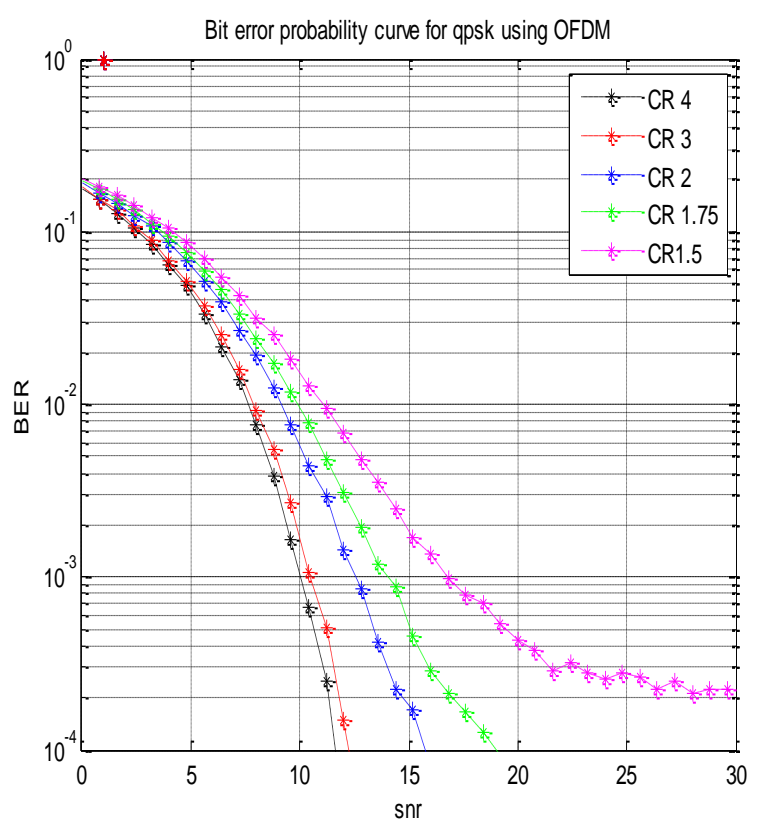

Fig 9: Comparison in BER for different CR

When CR decreases the PAPR improve on account of BER but as shown in figures $(8,9) \mathrm{CR}=3$ has batter PAPR with slightly degraded in BER compare with $\mathrm{CR}=4$ And $\mathrm{CR}=2$ has better than 3, 4 PAPR and acceptable BER degradation.

\section{CONCLUSIONS}

The hybrid PAPR has been proposed in this paper is better than just repeated clipping and frequency domain filtering or just SQRT companding. It can significantly reduce high peaks in the OFDM systems better than the both. At least 5 $\mathrm{dB}$ better than both types. But at the expense of increased complexity a little bit compares with just SQRT or just repeated clipping, but this technique still low complex compare with other PAPR techniques such as PTS, SLM, coding.

And PAPR can reduce depending on two parameters the oversampling and clipping ratio When CR is low PAPR reduce and the same thing for oversampling but on account BER and vice versa.

\section{REFERENCES}

[1] R. Van. Nee and R. Prasad, 2000. OFDM for Wireless Multimedia Communication. London: Artech house.

[2] IEEE Standard 802.11a, 1999. "Wireless LAN medium access control (MAC) and physical layer (PHY) specifications".

[3] IEEE Standard 802.16a, 2003. "Air interface for fixed broadband wireless access systems -Amendment 2: medium access control modifications and additional physical layer specifications for 2-11 GHz,"

[4] ITU-R, Report M.2134, 2008. Requirements related to technical performance for IMT-Advance Radio Interface.

[5] Hem Dutt Joshi, May 2012 "performance augmentation of OFDM system," Ph.D. dissertation, Jaypee Univ. of engineering and Technology, India. 
[6] Young Soo Cho, Jaekwon Kim, Won Young Yang and Chung G. Kahn, 2010. MIMO OFDM wireless communications with Matlab, Asia: John Wiley \& Sons.

[7] Gavin Hill, March 2011 "Peak Power Reduction in Orthogonal Frequency Division Multiplexing Transmitters," Ph.D. Thesis, Victoria University of Technology.

[8] Leonard J. Cimini, Jr., and Nelson R. Sollenberger Jun 1999. "Peak-to-average power ratio reduction of an OFDM signal using partial transmit sequences," Communications, 1999. ICC '99. 1999 IEEE International Conference on, vol.1, pp 511-515, 06-10.

[9] Sarita Singh Bhadhoria and S. Gupta, March 2013 "PAPR Reduction of OFDM Signals Using Selected Mapping Technique," Current Research in Engineering, Science and Technology (CREST) Journals, vol.1,no. 1, pp 34-37.

[10] M. Chauhan and A. Chobey, August 2012. "PAPR Reduction in OFDM system Using Tone Reservation Technique," International Journal of Computer Technology and Electronics Engineering (IJCTEE), vol. 2, no. 4, pp 57-60.

[11] J. Hou, C. Tellambura, and J. Ge June 2013, "Clipping Noise-based Tone Injection for PAPR Reduction in OFDM Systems," Communications (ICC), 2013 IEEE International Conference on, pp 5759 -5763, 9-13.

[12] M. K. Singh, S. Sharma, P. Singh, A. Gupta, S. K. Singh Aug 2012. "Different PAPR Reduction Techniques for Orthogonal Frequency Division Multiplexed Signals", International Journal of Electronics and Computer Science Engineering.
[13] S. H. Han and J. H. Lee April 2005. "An overview of peak-to-average power ratio reduction techniques for multicarrier transmission", Wireless Communications, IEEE ,vol.12, no: 2 ,pp 56-65.

[14] T. Jiang and Y. Wu, June 2008. "An overview: peak-toaverage power ratio reduction techniques for OFDM signals," IEEE Transactions on Broadcasting, vol. 54, no. 2, pp. 257- 268.

[15] Carole A. Devlin, Anding Zhu, and Thomas J. Brazil Jan. 2008. "Peak to Average Power Ratio Reduction Technique for OFDM Using Pilot Tones and Unused Carriers" Radio and Wireless Symposium, 2008 IEEE , pp $33-36,22-24$.

[16] Armstrong, J., February 2002. "Peak-to-average power reduction for OFDM by repeated clipping and frequency domain filtering" ELECTRONICS LErrERS, vol. 38 , no. 5, pp. 246- $247,28^{\text {th }}$.

[17] Armstrong, J., 2001 "New OFDM peak-to-average power reduction scheme," IEEE 53rd Vehicular Technology Conference, Vol. 1, no. Pp. 756-760.

[18] Wisam F. Al-Azzo, Borhanuddin M. Ali, Sabira Khatun, and S. M. Bilfagih, 2007. "Time domain statistical control for PAPR reduction in OFDM system", Proc. IEEE APCC 2007, Bangkok, Thailand, pp.141-144.

[19] Wisam F. Al-Azzo, Borhanuddin M. Ali, march 2011. "Adaptive Square-Rooting Companding Technique for PAPR Reduction in OFDM Systems", World Academy of Science, Engineering and Technology, Vol. 5, $20^{\text {th }}$.

[20] S. Haykin and M. Moher, , 2005. Modern Wireless Communications. Prentice Hall. 